\title{
An Efficient, Counter-Selection-Based Method for Prophage Curing in Pseudomonas aeruginosa Strains
}

\author{
Esther Shmidov ${ }^{1,2}$, Itzhak Zander ${ }^{1,2}$, Ilana Lebenthal-Loinger ${ }^{1}$, Sarit Karako-Lampert ${ }^{3}$ (D), Sivan Shoshani ${ }^{1,2}$ and \\ Ehud Banin 1,2,* \\ 1 The Mina and Everard Goodman Faculty of Life Sciences, Bar-Ilan University, Ramat-Gan 5290002, Israel; \\ Estern95@gmail.com (E.S.); itzhak.zan@gmail.com (I.Z.); ilana.loinger@biu.ac.il (I.L.-L.); \\ sivan.shoshani@biu.ac.il (S.S.) \\ 2 The Institute of Nanotechnology and Advanced Materials, Bar-Ilan University, Ramat Gan 5290002, Israel \\ 3 Scientific Equipment Center, The Mina \& Everard Goodman Faculty of Life Sciences Bar-Ilan University, \\ Ramat Gan 5290002, Israel; sarit.lampert@gmail.com \\ * Correspondence: Ehud.Banin@biu.ac.il
}

Citation: Shmidov, E.; Zander, I.; Lebenthal-Loinger, I.; Karako-Lampert, S.; Shoshani, S.; Banin, E. An Efficient, Counter-Selection-Based Method for Prophage Curing in Pseudomonas aeruginosa Strains. Viruses 2021, 13, 336. https://doi.org/10.3390/ v13020336

Academic Editor: Dann Turner

Received: 2 February 2021

Accepted: 18 February 2021

Published: 21 February 2021

Publisher's Note: MDPI stays neutral with regard to jurisdictional claims in published maps and institutional affiliations.

Copyright: (c) 2021 by the authors. Licensee MDPI, Basel, Switzerland. This article is an open access article distributed under the terms and conditions of the Creative Commons Attribution (CC BY) license (https:/ / creativecommons.org/licenses/by/ $4.0 /)$.

\begin{abstract}
Prophages are bacteriophages in the lysogenic state, where the viral genome is inserted within the bacterial chromosome. They contribute to strain genetic variability and can influence bacterial phenotypes. Prophages are highly abundant among the strains of the opportunistic pathogen Pseudomonas aeruginosa and were shown to confer specific traits that can promote strain pathogenicity. The main difficulty of studying those regions is the lack of a simple prophage-curing method for $P$. aeruginosa strains. In this study, we developed a novel, targeted-curing approach for prophages in $P$. aeruginosa. In the first step, we tagged the prophage for curing with an ampicillin resistance cassette $(a m p R)$ and further used this strain for the $s a c B$ counter-selection marker's temporal insertion into the prophage region. The sucrose counter-selection resulted in different variants when the prophagecured mutant is the sole variant that lost the $\operatorname{ampR}$ cassette. Next, we validated the targeted-curing with local PCR amplification and Whole Genome Sequencing. The application of the strategy resulted in high efficiency both for curing the Pf4 prophage of the laboratory wild-type (WT) strain PAO1 and for PR2 prophage from the clinical, hard to genetically manipulate, 39016 strain. We believe this method can support the research and growing interest in prophage biology in P. aeruginosa as well as additional Gram-negative bacteria.
\end{abstract}

Keywords: Pseudomonas aeruginosa; prophages; bacteriophages; curing; counter-selection; lysogen

\section{Introduction}

Pseudomonas aeruginosa is a Gram-negative, rod-shaped bacterium with a single flagellum. It is an opportunistic pathogen of plants, nematodes, insects, animals, and humans [1]. It can cause a wide range of acute and chronic infections, such as in severe wounds or burns, and chronic lung infections in cystic fibrosis (CF) patients [2,3]. This is enhanced by the bacterium's low susceptibility to various antimicrobial substances, making most of the infections difficult to treat and life threatening [4,5]. The genetic variability among $P$. aeruginosa isolates is relatively low; different $P$. aeruginosa strains share a conserved "core genome" and differ in the variable "accessory segments" [6]. The accessory genome in $P$. aeruginosa consists mainly of genomic islands and mobile genetic elements (MGEs) such as plasmids, transposons, and prophages-like elements (temperate bacteriophages).

Bacteriophages (phages) are viruses that infect bacteria. They are highly abundant, rapidly spreading, and very diverse biological entities. Phage infections divide into productive lytic or lysogenic (temperate), depending on several factors such as host genetics, phage genetics, environmental conditions, and phage concentration [7]. In the lytic life cycle, phage infection is usually followed by intensive viral DNA replication, eventually leading to a high rate of new phage production and bacterial host cell lysis. In the lysogenic 
life cycle, the viral genome is integrated into the bacterial genome as a prophage and further replicates throughout bacterial cell division. A temperate phage can be triggered into the lytic cycle (excision event) spontaneously at a low frequency or by an external stressor such as bacterial DNA damage and physical or chemical factors [7]. The integration of a lysogenic phage into the bacterial genome can influence bacterial phenotypes by providing new traits such as antibiotic resistance, metabolic factors, as well as immunity against specific viral re-infections [7,8]. In some pathogenic bacterial species, prophages encode virulence factors and mediate bacterial adaptation in ecological niches [9]. As mobile elements, prophages are an essential tool for horizontal gene transfer between bacteria and, among other factors, contribute to strain genetic variability.

Most $P$. aeruginosa strains were identified to contain at least one prophage-like element; some are poly-lysogens, harboring several prophages in their genome [10,11]. Lysogenic phages in P. aeruginosa have been shown to confer selective beneficial traits, such as $\mathrm{O}$ antigen conversion, biofilm development, and virulence [12-14].

The PAO1 laboratory strain contains Pf1-like filamentous phage (Pf4) as a prophage that plays a role in small colony variant (SCV) formation during P. aeruginosa biofilm development and has a symbiotic role in bacterial biofilm formation $[15,16]$. Moreover, 39016 is a clinical isolate of $P$. aeruginosa that phenotypically differs from the laboratory wild-type (WT) strain PAO1; it exhibits a slower growth rate, reduced motility, and higher biofilm production levels [17]. The prophage-region screening of the published genome of the 39016 strain revealed that it contains five different prophage-like regions in its genome, and four of them are predicted to be intact and able to create infectious virions. As 39016 and PAO1 share the high conserved "core genome" and considering the phenotypic variation among these two strains, the prophage regions of 39016 are likely to influence the bacterial physiology and virulence.

A comparison of isogenic strains that differ only in their prophage region can contribute to understanding the biological role of a specific phage to the observed traits, the overall prophages contribution, and the cross-regulation between the prophages in polylysogenic strains. The main difficulty for this type of study is the lack of a simple and efficient experimental method for curing prophages in P. aeruginosa strains.

Previously described prophage curing methods, both for Gram-negative and Grampositive bacteria, mostly relied on promoting the phage loss with the use of DNA-damaging reagents and the activation of the SOS-response and thus potentially causing a variety of genomic mutations in the bacterial genome, which might result in further variation of the phenotypes $[18,19]$. Here, we introduce a simple, non-SOS based, highly efficient prophage curing method in P. aeruginosa, using the Gram-negative popular counter-selection marker $s a c B$ [20]. We applied the prophage curing method on Pf4 from the WT strain PAO1 and a prophage from the clinical isolate 39016, herein termed PR2. The method can theoretically be applied for all the Gram-negative bacteria containing an intact, excisable prophage in their genome, allowing a better investigation of the prophages and their contribution to bacterial physiology and virulence.

\section{Materials and Methods}

\subsection{Bacterial Strains, Plasmids and Growth Media}

The bacterial strains and plasmids used in this study are listed in Table 1. Primers are listed in Supplementary Table S1. All strains were grown on LB (Luria-Bertani broth, Difco, Sparks, MD, USA) at $37^{\circ} \mathrm{C}$ unless otherwise specified. For the insertion processes, the following media were used: Vogel Bonner Minimal Medium (VBMM) [21], Pseudomonas Isolation Agar (PIA, Difco, Sparks, MD, USA), and No salt Luria-Bertani (NSLB) $+10 \%$ sucrose. For the plasmid curing assay and DH5 $\alpha$ heat shock, BHI (Brain Heart Infusion broth, Difco, Sparks, MD, USA) media was used. Antibiotic concentrations used in this study were $300 \mu \mathrm{g} / \mathrm{mL}$ carbenicillin $(\mathrm{Crb})$ and $50 \mu \mathrm{g} / \mathrm{mL}$ gentamicin $(\mathrm{Gm})$ for P. aeruginosa and $100 \mu \mathrm{g} / \mathrm{mL}$ ampicillin (Amp) and $30 \mu \mathrm{g} / \mathrm{mL}$ Gm for Escherichia coli. 
Table 1. Strains and plasmids used in the study.

\begin{tabular}{|c|c|c|}
\hline Strain or plasmid & Description & Source \\
\hline \multicolumn{3}{|c|}{ P. aeruginosa strains } \\
\hline PA01 & WT & [22] \\
\hline PAO1 $\Delta$ Pf4 & PAO1, $\triangle \mathrm{Pf} 4$ & This study \\
\hline PAO1 $\Delta p f i T$ & PAO1, $\triangle \mathrm{PAO} 729:: \mathrm{Crb}^{\mathrm{r}}$ & {$[23]$} \\
\hline 39016 & $\begin{array}{c}\text { LMG 27,647 P. aeruginosa (Schoeter 1872) } \\
\text { migula } 1900 \text { AL }\end{array}$ & $\begin{array}{l}\text { BCCM-biological origin: } \\
\text { keratitis patients }\end{array}$ \\
\hline 39016/PR2_ampR & 39016, $\Delta$ PA39016_000100015::Crb ${ }^{\mathrm{r}}$ & This study \\
\hline $30916 \Delta \mathrm{PR} 2 / \mathrm{Gm}^{\mathrm{r}}$ & $39016, \triangle \mathrm{PR} 2:: \mathrm{Gm}^{\mathrm{r}}$ & This study \\
\hline $30916 \Delta \mathrm{PR} 2$ & $39016, \Delta \mathrm{PR} 2$ & This study \\
\hline \multicolumn{3}{|c|}{ E. coli strains } \\
\hline DH5 $\alpha$ & $\begin{array}{l}\text { F- } \Phi 80 \text { lacZ } \Delta \text { M15 } \Delta(\text { lacZYA-argF) U169 } \\
\text { recA1 endA1 hsdR17 (rK-, mK+) phoA } \\
\text { supE44 } \lambda \text { - thi-1 gyrA96 relA1. }\end{array}$ & Bio-Lab \\
\hline S17 & $\begin{array}{l}\text { E. coli S17 thi, pro, hsdR, recA::RP4 } \\
\text {-2-Tc::Mu aphA::Tn7, } \lambda \text {-pir, Smr, Tpr }\end{array}$ & [24] \\
\hline \multicolumn{3}{|c|}{ Plasmids } \\
\hline pUCP18-Ap & $\begin{array}{l}\mathrm{Crb}^{\mathrm{r}} \text { (for P. aeruginosa), } \mathrm{Amp}^{\mathrm{r}} \text { (for E. coli), } \\
\text { overexpression plasmid, lacZ promoter } \\
\mathrm{Gm}^{\mathrm{r}} \text { and } \mathrm{Cm}^{\mathrm{r}}, \text { pEX18Gm containing a }\end{array}$ & [25] \\
\hline pDONRPEX18Gm & $\begin{array}{l}\text { HindIII flanked, attP cloning site from } \\
\text { pDONR201 }\end{array}$ & [21] \\
\hline pDONER/AmpRin_PR2 & $\begin{array}{c}\mathrm{Cm}^{\mathrm{r}} \text { and } \mathrm{Gm}^{\mathrm{r}}, \text { PA39016_000100015 } \\
\text { upstream and downstream fragments } \\
\text { with AmpR cassette inserted into } \\
\text { pDONRPEX18Gm by Gateway } \\
\text { recombination }\end{array}$ & This study \\
\hline pDONER/PR2_SacB & $\begin{array}{c}\mathrm{Cm}^{\mathrm{r}} \text { and } \mathrm{Gm}^{\mathrm{r}} \text {, PA39016_000100043 and } \\
\text { PA39016_000100035 fragments inserted } \\
\text { into pDONRPEX18Gm by Gateway } \\
\text { recombination }\end{array}$ & This study \\
\hline pDONER/Pf4_SacB & $\begin{array}{c}\mathrm{Cm}^{\mathrm{r}} \text { and } \mathrm{Gm}^{\mathrm{r}}, \text { PA0725 upstream and } \\
\text { downstream fragments inserted into } \\
\text { pDONRPEX18Gm by Gateway } \\
\text { recombination }\end{array}$ & This study \\
\hline
\end{tabular}

\subsection{DNA Manipulation and Plasmid Construction}

Genomic extraction was performed using the DNeasy Blood \& Cell Culture DNA Kit (Qiagen, Hilden, Germany). For DNA fragment amplification, Phusion High-Fidelity DNA Polymerase (Thermo) was used. DNA fragments upstream and downstream to the deletion/insertion region were amplified from genomic DNA using PCR. The amplified inserts were cleaned using NucleoSpin Gel and PCR Clean-up (MACHEREY-NAGEL). $a m p R$ cassette was amplified from the plasmid pUCP18-Ap using PCR (primers ampR_F and ampR_R). The segments were inserted into PEX18Gm-GW plasmid by recombination (Gateway recombination system and BP Clonase enzyme, Invitrogen, Carlsbad, CA, USA). The DNA Polymerase ReddyMix PCR Kit and M13_F and M13_R universal primers were used to verify successful plasmid transformations. The plasmids were extracted with QIAprep spin miniprep kit (QIAGEN, Hilden, Germany) and sequenced. All of these processes were carried out according to the manufacturer's instructions.

\subsection{Insertion of ampR into Phage Region}

The PAO1/Pf4_ampR ( $\Delta p f i T)$ and 39016/PR2_ampR strains construction was carried out as previously described [21] with minor changes using the $a m p R$ cassette. In PR2 
prophage, the $a m p R$ cassette was inserted instead of the PA39016_000100015 gene. For Pf4 phage curing, the $a m p R$ gene was inserted inside the Pf4 locus at ORF PA0729 [23].

\subsection{Prophage Curing and Selection for Phage-Cured Mutants}

The $a m p R$-containing strains were further inserted with $s a c B$ into the phage region, as previously described with some process adaptation changes [21]. Briefly, merodiploids with the pDONER/Pf4_SacB or the pDONER/PR2_SacB plasmid undergo site-specific integration into the Pf4 and PR2, respectively, by vector-encoded homologous sequences of prophage region. The merodiploids were streaked on NSLB plates containing $10 \%$ sucrose and were incubated for $24 \mathrm{~h}$ at $30^{\circ} \mathrm{C}$. Single colonies isolated on the sucrose agar were taken and transferred to PIA, LB Gm, and LB Crb plates and incubated overnight. Positive $P$. aeruginosa colonies that contained the desired deletion grew on PIA plates, but neither on LB Gm nor on LB Crb containing plates. The mutants were verified for complete loss of the phage by PCR for prophage genes and the intact bacterial attachment site attB.

\subsection{Plasmid Curing}

For recombinant plasmid curing, the $39016 \Delta \mathrm{PR} 2 / \mathrm{Gm}^{\mathrm{r}}$ culture was incubated overnight in BHI at $42{ }^{\circ} \mathrm{C}$ with shaking and then streaked overnight on LB plate. Single colonies isolated on LB plates were then transferred to the LB and LB Gm plates for Gm-sensitive variant identification.

\subsection{Phage Extraction}

LB $(2 \mathrm{~mL})$ was inoculated with bacterial strain and incubated overnight. The next day, the bacteria were diluted 1:50 with medium to a final volume of $4 \mathrm{~mL}$ and incubated for $3 \mathrm{~h}$. Then, $1 \mathrm{~mL}$ of bacteria was centrifuged at $14,000 \times g$ for $2 \mathrm{~min}$, and $900 \mu \mathrm{L}$ of the supernatant was filtered using a $0.45 \mu \mathrm{m}$ filter (Whatman, Maidstone, Kent, UK).

\subsection{Plaque Assay}

LB $(2 \mathrm{~mL})$ was inoculated with recipient strain and incubated overnight with shaking. The next day, bacteria were diluted 1:50 with medium to a final volume of $2 \mathrm{~mL}$ and incubated with shaking for $2 \mathrm{~h}$. Further, $100 \mu \mathrm{L}$ of the bacteria was transferred into $5 \mathrm{~mL}$ heated $\left(50{ }^{\circ} \mathrm{C}\right) \mathrm{LB}$ with $0.5 \%$ agar, and then gently mixed and poured onto the surface of a $1.5 \%$ LB-agar plate. Serial dilutions (1:10) of the extracted phage stock were made, droplets of $2 \mu \mathrm{L}$ were spotted on to the top-agar layer. The plate was incubated overnight until plaques were formed.

\subsection{Whole-Genome Sequencing}

DNA was extracted from cell pellets using the DNeasy Blood \& Tissue Kit (Qiagen), and DNA quality was evaluated by gel electrophoresis. The library was constructed with NEBNext Ultra II FS DNA Library Prep Kit for Illumina (NEB, UK) according to the manufacturer's instructions using $500 \mathrm{ng}$ as the starting material. DNA was fragmented $20 \mathrm{~min}$ with three cycles of PCR. The final quality was evaluated by TapeStation High Sensitivity D1000 Assay (Agilent Technologies, CA, USA). Sequencing was performed based on Qubit values and loaded onto an Illumina MiSeq using the MiSeq Micro 150× 2 kit (Illumina, CA, USA).

\subsection{Genome Assembly and Sequence Analysis}

The MiSeq sequencing resulted in 1.13-2.87 million paired-end reads per strain. FastQC (v0.11.2) (https:/ / www.bioinformatics.babraham.ac.uk/projects/fastqc(accessed on 11 February 2021)) was used to assess the quality of the raw reads. For each strain de novo assembly was performed by SPAdes (v3.13.0) [26] with parameters -k 21,33,55,77,99, 127 -careful. QUAST (v5.0.2) [27] was used for quality assessment of the genome assemblies. Contigs with a minimal length of 500 bases from the assemblies of P. aeruginosa 39016 (WT and mutant) and P. aeruginosa PAO1 (WT and mutant) were reordered according to reference genomes (NCBI Reference Sequence NZ_CM001020.1 and NC_002516.2, respec- 
tively) using Mauve aligner [28,29]. Regions of prophage insertion were determined using PHASTER [30]. BLAST Ring Image Generator (BRIG) (https:/ / sourceforge.net/ projects / brig/ (accessed on 11 February 2021)) [31], and Mauve [28] were used to compare the WT and mutant to the reference genome.

\section{Results}

\subsection{The Targeted Curing Principle}

The overall principle of the curing strategy is presented in Figure 1. The first step was to tag the targeted prophage with an $a m p R$ cassette insertion into the phage region using an allelic replacement technique. After creating the ampicillin-resistant strain, it was further used for $s a c B$ insertion by utilizing the site-specific integration step of the gentamicin resistance marker (aacC1) and $s a c B$ containing plasmid backbone in the allelic replacement method. Homologous regions were used to direct the backbone integration into the prophage, and in this way, a temperate $a m p R / s a c B / a a c C 1$ containing-form of the prophage was created (see scheme in Figure 1). To push toward either insertion or the desired prophage curing, the merodiploids were streaked on sucrose plates. The sucrose counter-selection resulted in either no change in the ampR-inserted prophage (Figure 1A), integration-site deletion mutants (Figure 1B), sacB mutants that survived the sucrose selection (Figure 1C), or the whole prophage-cured variant (Figure 1D). Out of all these options, the prophage-cured mutant is the sole Crb-sensitive variant (Figure 1). Such a system makes it possible to select for rare colonies that lost the ampR-inserted prophage. The separation of the two steps was to assure insertion distance between the counterselection and the positive-selection markers to avoid false-positive results in variants that lost all the markers but not the whole prophage.

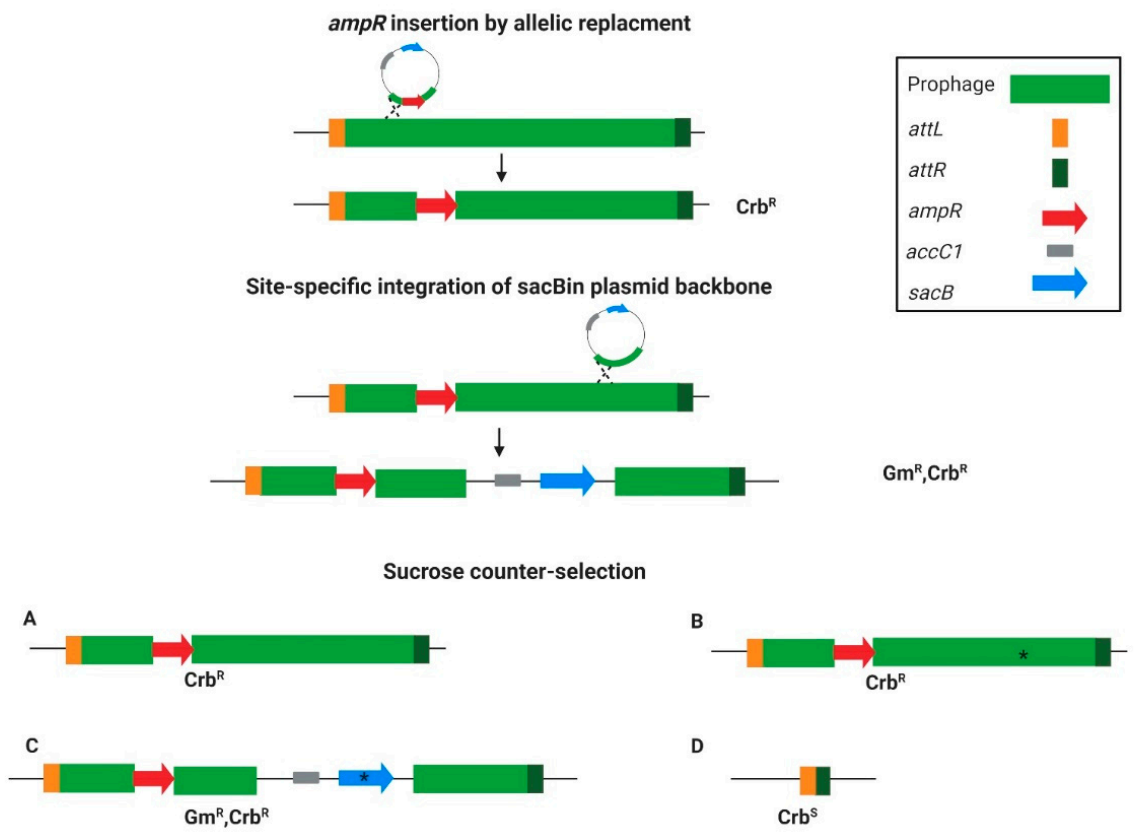

Figure 1. The targeted curing method principle. The prophage is bordered by the right attachment site attR (dark green) and the left attachment site attL (orange). The first step is $a m p R$ (red) insertion for prophage tagging. The second step is the integration of the plasmid backbone, containing accC1 (gray) and $s a c B$ (light blue) into the prophage region via homologous recombination. Next, the temporal tagged prophage with the integrated $s a c B$ undergoes counter-selection by growing on the sucrose-containing growth medium. The counter-selection outcome can be either (A) unchanged tagged prophage, (B) integration site deletion (marked with an asterisk), (C) sacB mutated variant (asterisk), or (D) prophage curing. Notably, only option (D) would be Crb sensitive. 


\subsection{Pf4 Phage of PAO1 Curing}

The curing of Pf4 prophage of the PAO1 strain was carried out to demonstrate the efficiency of the targeted curing method.

First, PAO1/Pf4_ampR $(\triangle p f i T)$ mutant was created by inserting the ampR cassette instead of the PAO729 gene, which encodes for the PfiT toxin protein from the toxinantitoxin (TA) pfiT/pfiA system. The insertion did not alter the bacterial growth compared to WT PAO1, and the strain still showed spontaneous phage loss [23].

To further integrate the $s a c B$-containing plasmid backbone, the upstream and downstream homologous sequences of the PA0725 gene were used to direct the insertion into the prophage and create the transient $a m p R / s a c B / a c c C 1$ containing Pf4-containing prophage. The counter-selection on sucrose-containing medium resulted in a high yield; $100 \%$ of the analyzed colonies (40/40) were both $\mathrm{Crb}$ - and $\mathrm{Gm}$-sensitive, indicating a potential phage loss.

Complete loss of the Pf4 prophage and the circular replicative form (RF) DNA was confirmed for this mutant by PCR amplification around the attB site of Pf4, the attR site for the integrated form, and the phage attachment site (attP) for the RF form of Pf4 phage (Figure 2A-C). One such mutant was randomly chosen and designated as $\triangle \mathrm{Pf} 4$ strain. As the WT PAO1 strain exhibits immunity properties against Pf4 re-infection, the $\Delta \mathrm{Pf} 4$ strain was tested for immunity loss. The cured strain infection with Pf4 phages, extracted from WT PAO1, resulted in plaque formation and bacterial susceptibility, unlike the WT strain (Figure 2D).

A
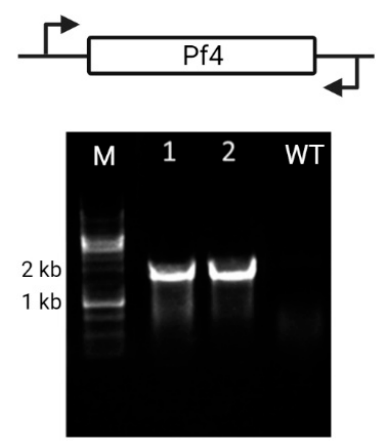

C

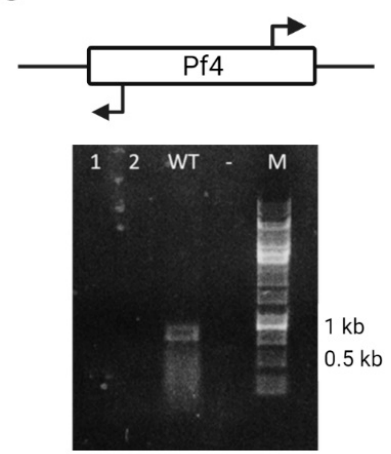

B
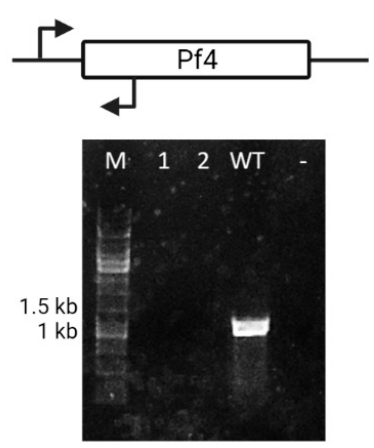

D

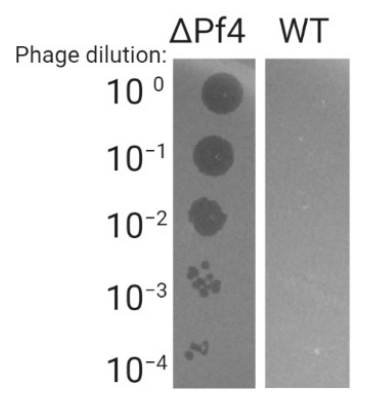

Figure 2. Pf4 curing verification. (A-C) Curing verification by PCR, Lanes 1-2 represent the DNA of randomly picked Crb-sensitive colonies, and lane 3 represents the WT PAO1 for control. (A) Amplification of $1800 \mathrm{bp}$ around the Pf4 attB site. (B) Amplification of $1000 \mathrm{bp}$ around the Pf 4 attR site that can only be amplified in the integrated form. (C) Amplification of $750 \mathrm{bp}$ around the Pf 4 attP site that can only be amplified in the RF form. (D) Curing verification by plaque assay, serial dilutions of Pf4 phage are extracted from WT PAO1 strain used to infect WT PAO1 and $\triangle \mathrm{Pf} 4$ strain. 
To assure the genome integrity of this strain and to prove once more the phage loss, whole-genome sequencing was performed to the cured strain, and the resulting assembly was aligned to the PAO1 WT sequence. The genome analysis revealed that the WT strain assembly covers $99.284 \%$ of the reference genome and that besides the Pf4 region, no additional significant insertions or deletions were detected in the mutant strain (Figure 3).

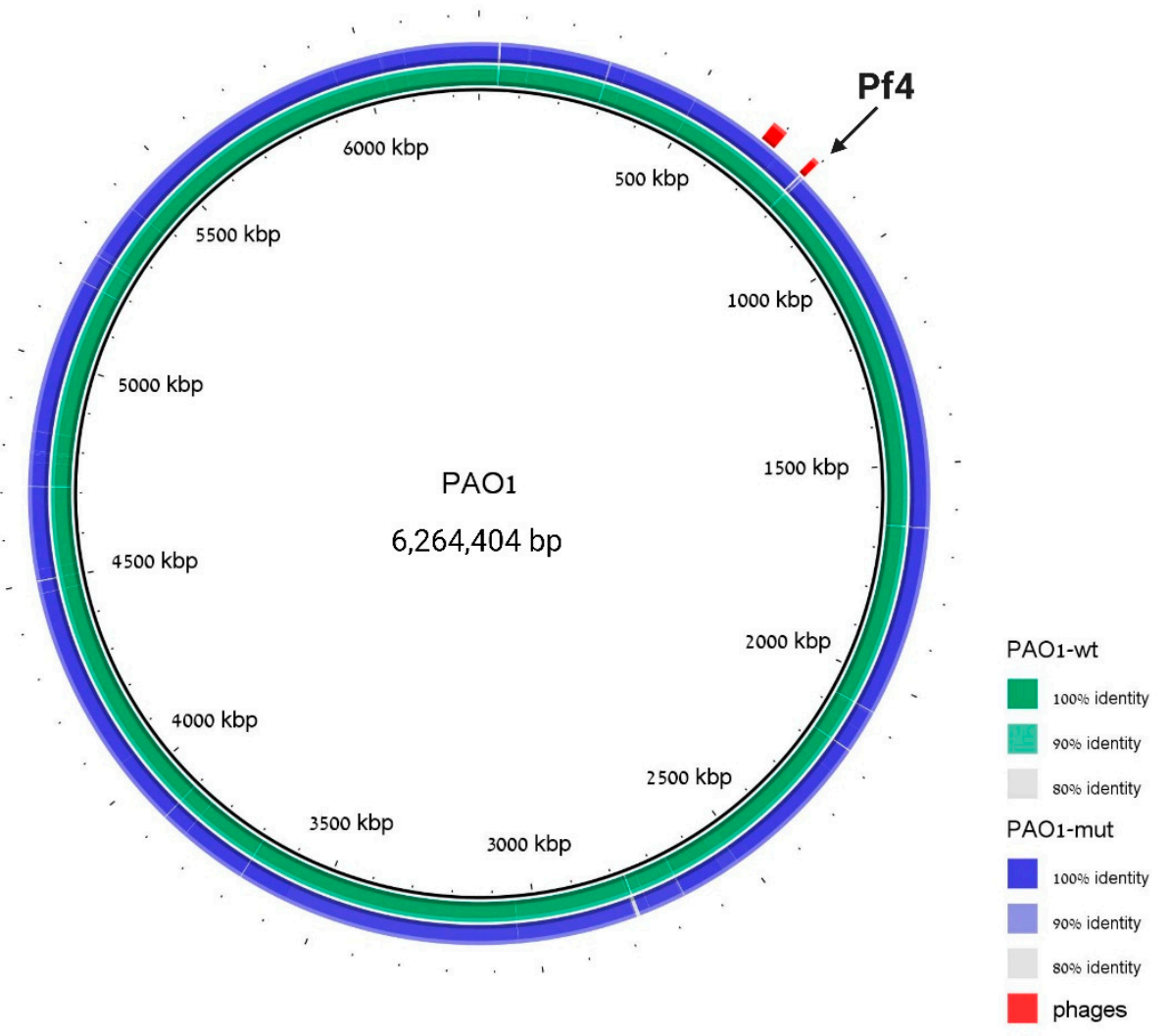

Figure 3. The $\triangle \mathrm{Pf} 4$ strain differs from WT in the Pf4 region. Genomic alignment with PAO1 strain is used as a reference, PAO1 WT sequence assembly is indicated in green, and $\triangle \mathrm{Pf} 4$ strain sequence assembly is indicated in blue. Prophage regions are labeled in red; coordinates are taken from PHASTER analysis to the reference PAO1 strain. The cured region is marked with an arrow. We note that there is a difference between our laboratory WT strain and the reference strain around region $2800 \mathrm{bp}$, but this difference is identical in the Pf4-mutant strain.

\subsection{PR2 Phage of 39016 Curing}

Clinical isolates of P. aeruginosa are less attenuated than the WT PAO1 strain; thus, the genetic manipulations are limited and challenging in those isolates. In order to demonstrate the efficiency of our method for a clinical isolate, the 39016 strain of P. aeruginosa was tested for curing its second prophage (PR2).

First, 39016/PR2_ampR mutant was created by inserting the $\operatorname{ampR}$ cassette instead of the PA39016_000100015 gene (hypothetical protein, herein termed as AmpRin region). Next, to assure that the insertion did not affect the excision ability of PR2, a comparison of the infectivity of phages produced by WT 39016 or by 39016/PR2_ampR strain has been performed. The results showed that the cassette's insertion did not impair the bacterial growth or the infectious phage production of the 39016 strain (Figure S1).

Further $s a c B$ integration was directed by homologous sequences upstream and downstream to the intragenic region of PR2 located between the PA39016_000100034 and PA39016_000100035 genes (SacBin region). After the sucrose counter-selection, the antibiotic screen revealed approximately $70 \%(57 / 80)$ Crb-sensitive colonies that were also $\mathrm{Gm}$ resistant. Prophage gene amplification indicated that the phage was lost (Figure 4A); however, the SacBin region was still present in the cured strain (Figure S2) indicating some 
recombinant plasmid has been created in the process that contains the accC1 marker and the SacBin region; this strain was named " $\triangle \mathrm{PR} 2 / \mathrm{Gm}^{\mathrm{R}}$." In order to cure the recombinant plasmid, we applied a temperature-based plasmid curing assay on $\triangle \mathrm{PR} 2 / \mathrm{Gm}^{\mathrm{R}}$ strain. $\mathrm{PCR}$ confirmed the resulting $\mathrm{Gm}$-sensitive colonies for the SacBin region loss and attB region amplification for the complete phage loss (Figure 4B). One such mutant was chosen for study and designated as $\triangle \mathrm{PR} 2$ strain.

A

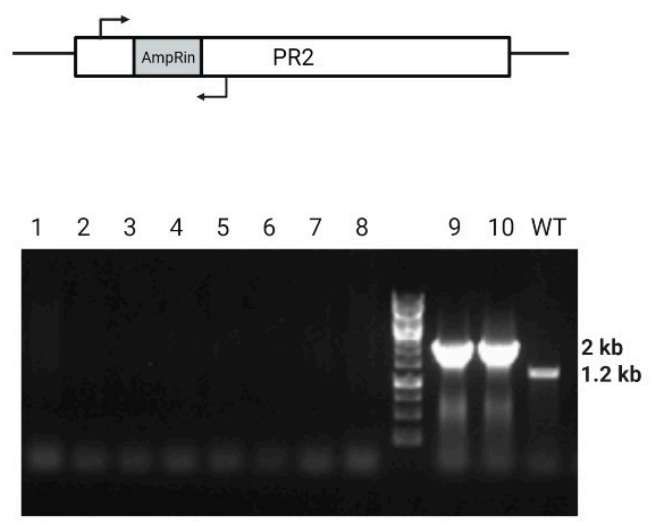

B
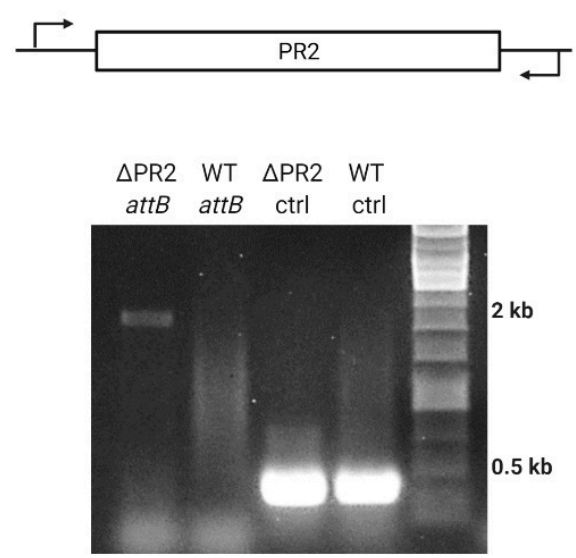

Figure 4. PR2 curing verification by PCR. (A) AmpRin region amplification results either in null, $2000 \mathrm{bp}$, or $1200 \mathrm{bp}$ in the cured, ampR-containing, or WT strain, respectively; lanes 1-8 represent randomly picked Crb-sensitive colonies, lanes 9-10 represent Crb-resistant colonies, and the WT 39016 is for positive control. (B) Amplification of 2000 bp around the PR2 attB site of $\triangle$ PR2 and WT 39016, and positive control (ctrl) amplification of 324 bp PA39016_100004 external gene.

To verify that both the prophage-curing and the plasmid-curing processes did not result in a high mutation rate in the $\triangle \mathrm{PR} 2$ strain, the cured strain genome was sequenced and aligned to the WT 39016 sequence. The genome analysis revealed that PR2 was missing in the cured strain, and no additional significant insertions or deletions were detected (Figure 5). Moreover, PHASTER [30] analysis of the assembled genome of $\triangle \mathrm{PR} 2$ revealed that the cured strain differs from the WT 39016 in its prophage content solely in the second phage; the curing process did not affect all the other phages of 39016.

Unfortunately, the attempts to use the cured $\triangle \mathrm{PR} 2$ strain as a host by infecting with phages extracted from WT 39016 strain had failed, suggesting some cross-immunity between PR2 and the other prophages in 39016. However, as indicated by PCR amplification of the extracted intact phages, while successful PR2 virion production was observed in the WT strain, it is not produced by the cured strain (Figure S3). 


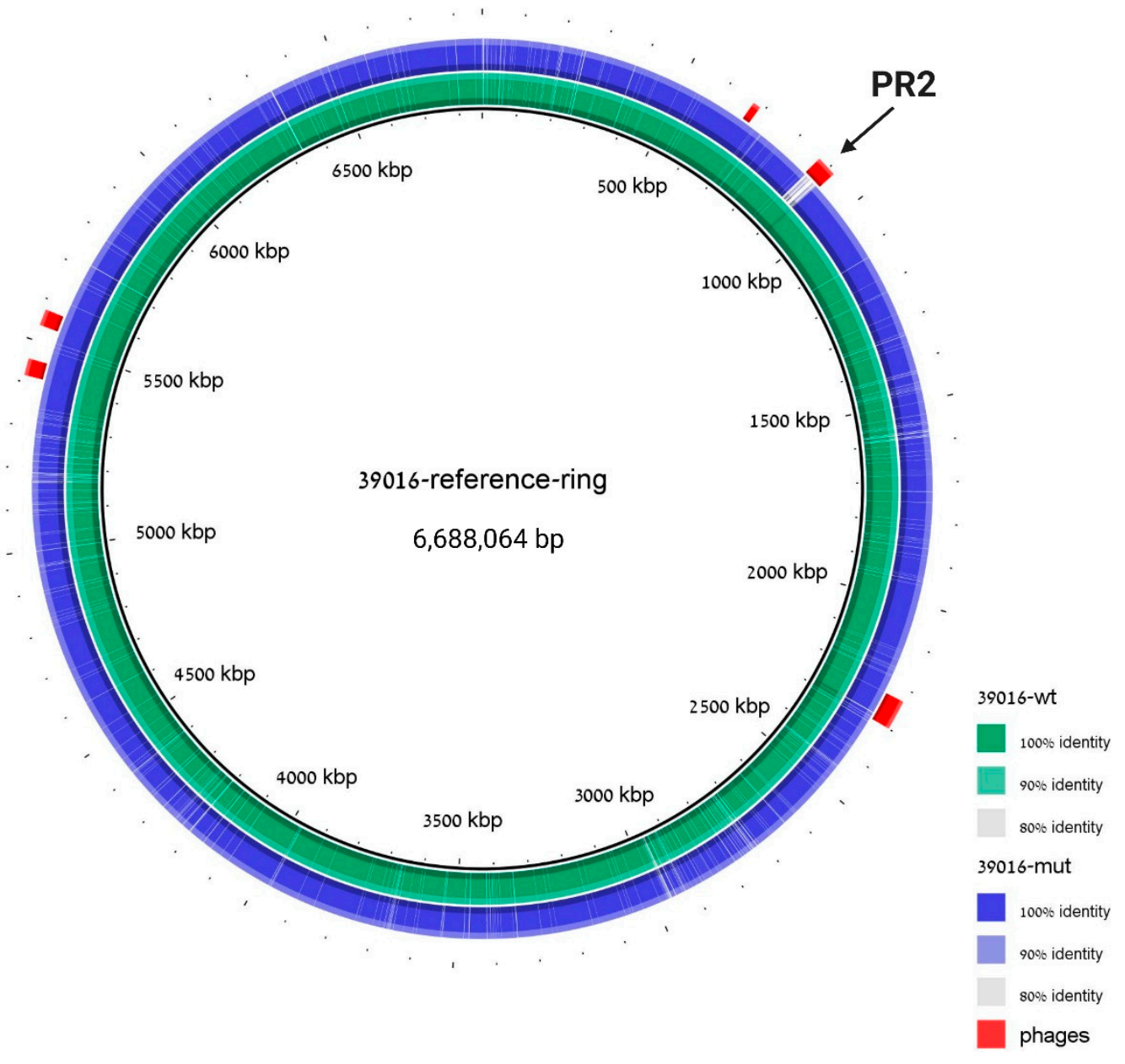

Figure 5. The $\triangle \mathrm{PR} 2$ strain differs from WT in the PR2 region solely. Genomic alignment with 39016 strain is used as a reference, 39016 WT sequence assembly is indicated in green, and $\triangle$ PR2 strain sequence assembly is indicated in blue. Prophage regions are labeled in red; coordinates are taken from PHASTER analysis to the reference 39016 strain. The cured region is marked with an arrow.

\section{Discussion}

In this study, we provide a simple counter-selection-based curing methodology. The approach was proven successful for both the laboratory PAO1 WT strain and the clinical, hard to genetically manipulate, 39016 strain. The guiding principles of the targeted method were first to avoid DNA damaging as in the usage of SOS response promoting agents, and second, to cure a specific prophage without influencing the other prophages in the genome of the poly-lysogenic strains.

The strategy for prophage targeted curing from poly-lysogenic strains should take into the similarity of the different prophages. For poly-lysogenic strains that carry different prophages, there is no need for a spatial approach; as we have shown with 39016, the other prophages are not affected by the curing process. For strains that carry highly similar prophages, the tagging step should be directed to a unique region or a low-similarity region within the prophage. PAO1 is an example of such a ploy-lysogenic strain as it carries two highly similar prophages in its genome; Pf4 and Pf6 prophages [32]. We achieved 100\% curing by focusing on the unique region of Pf 4 for the ampR insertion step to assure proper prophage-tagging, and the second step was directed into a common region with high similarity for both Pf6 and Pf4. For strains that carry multiple copies of the same prophage, it should be recommended to sequence properly following the tagging step to identify which of the prophages was eventually targeted to be cured in the second step. 
The use of a counter-selection marker for prophage curing has been described before for Streptococcus pyogenes [33]. The method is based on first creating a streptomycinresistant strain that is mutated in its $r p s L$ gene, and further insertion of other antibiotic resistance cassette and the rpsL WT allele for counter-selection by streptomycin. The difficulty of using such an approach in P. aeruginosa is mainly because it does not harbor streptomycin resistance through genomic mutations in the rpsL gene [34], so the counterselection marker is not suitable in this case. Furthermore, the resistant mutant creation process by genomic mutation might disrupt the genomic integrity and cause additional, uncontrolled mutations. Another paper described a different counter-selection-based curing technique in Vibrio natriegens by inserting an inducible $c c d B$ toxin into the phage region and further counter-selection by toxin induction [34]. The usage of $c c d B$ toxin or other toxins from TA modules might be problematic for targeted curing based on the assumption that the toxins might induce other prophages in the genome regardless of the curing process [23]. Our approach avoids such problems.

It is important to note that the curing process of PR2 from 39016 had, in addition to the targeted curing process, an additional plasmid curing step to remove the recombinant plasmid produced in the curing process. The creation of the recombinant plasmid might have occurred due to the conservation of an oriT, the atcc 1 cassette, and some specific, important regions of the prophage. A similar case was also reported in the curing of specific prophage in S. pyogenes. The produced recombinant plasmid in the reported case was related to the prophages' cross-regulatory properties. When attempting to cure the same phage, in the background of other cured prophages, the recombinant plasmid was not produced [33].

The curing process of Pf4 from PAO1 was very efficient, with a high yield of cured mutants that can serve as a surrogate for infection from PAO1-produced phages. The high yield might be related to the fact that the first curing step included the deletion of the pfiT toxin gene; in other cases, it might be less efficient due to the addiction mediated by the stable toxin that will promote killing in the cured, antitoxin free variants. Thus, as part of the curing assay, it is recommended to initially scan for TA systems in the specific prophage sequence and target the toxin in the $a m p R$ insertion step.

Using the double-stage approach, we managed to overcome the difficulty with the limited counter-selection available markers for $P$. aeruginosa, when in the first stage, the $s a c B$ marker was used for plasmid-backbone removal, and then, the same marker was utilized to select for complete phage loss from the curing step. It is important to note that the method can potentially be applied to other mobile genetic elements and stable-plasmids that are commonly associated with bacterial virulence $[35,36]$.

The principle of the method and the high observed efficiency gives a better understanding of prophage-host interactions. The prophage-excision event mostly attributed to the phage disadvantage for staying integrated [37] or regulated by the host for a temporary extra-chromosomal state (active lysogeny) [38], but here, we showed that under certain conditions, when the prophage's contents endanger the host, it would prefer to lose the whole prophage rather than the specific risk-containing region. This fact emphasizes the need for curing rather than deletion methods for such regions.

Supplementary Materials: The following are available online at https: / www.mdpi.com/1999-4 915/13/2/336/s1, Supplementary methods, Table S1: Primers used in the study, Figure S1: ampR insertion into the PR2 prophage did not alter the bacterial growth and PAO1-infectious phages production, Figure S2: The SacBin region presents in PR2-cured colonies. Figure S3: PR2 phages are not produced in the cured strain.

Author Contributions: Conceptualization, E.S., I.Z., and E.B.; methodology, E.S, I.Z., and S.K.-L. data analysis, E.S. and I.L.-L.; writing-original draft preparation, E.S.; writing-review and editing, E.S., S.S., and E.B.; supervision, E.B.; funding acquisition, E.B. All authors have read and agreed to the published version of the manuscript. 
Funding: This work is part of the Ph.D. thesis of E.S. Partial funding for this work was through the Dyna and Fala Weinstock Foundation to E.B., the President's Scholarships, and the Merit-Based Scholarships at the Institute of Nanotechnology of Bar-Ilan University for E.S.

Institutional Review Board Statement: Not applicable.

Informed Consent Statement: Not applicable.

Data Availability Statement: Not applicable.

Acknowledgments: We thank Yigal Brochin for editing the article.

Conflicts of Interest: The authors declare no conflict of interest.

\section{References}

1. Pirnay, J.P.; Bilocq, F.; Pot, B.; Cornelis, P.; Zizi, M.; Van Eldere, J.; Deschaght, P.; Vaneechoutte, M.; Jennes, S.; Pitt, T.; et al. Pseudomonas aeruginosa Population Structure Revisited. PLoS ONE 2009, 4, e7740. [CrossRef] [PubMed]

2. Mathee, K.; Lory, S.; Narasimhan, G.; Valdes, C.; Qiu, X.; Matewish, J.M.; Koehrsen, M.; Rokas, A.; Yandava, C.N.; Engels, R.; et al. Dynamics of Pseudomonas aeruginosa Genome Evolution. Proc. Natl. Acad. Sci. USA 2008, 105, 3100-3105. [CrossRef]

3. James, C.E.; Fothergill, J.L.; Kalwij, H.; Hall, A.J.; Cottell, J.; Brockhurst, M.A.; Winstanley, C. Differential Infection Properties of Three Inducible Prophages from an Epidemic Strain of Pseudomonas aeruginosa. BMC Microbiol. 2012, 12, 216. [CrossRef] [PubMed]

4. Aloush, V.; Navon-Venezia, S.; Seigman-Igra, Y.; Cabili, S.; Carmeli, Y. Multidrug-Resistant Pseudomonas aeruginosa: Risk Factors and Clinical Impact. Antimicrob. Agents Chemother. 2006, 50, 43-48. [CrossRef] [PubMed]

5. Gellatly, S.L.; Hancock, R.E.W. Pseudomonas aeruginosa: New Insights into Pathogenesis and Host Defenses. Pathog. Dis. 2013, 67, 159-173. [CrossRef] [PubMed]

6. Wiehlmann, L.; Wagner, G.; Cramer, N.; Siebert, B.; Gudowius, P.; Morales, G.; Kohler, T.; van Delden, C.; Weinel, C.; Slickers, P.; et al. Population Structure of Pseudomonas aeruginosa. Proc. Natl. Acad. Sci. USA 2007, 104, 8101-8106. [CrossRef] [PubMed]

7. Payet, J.P.; Suttle, C.A. To Kill or Not to Kill: The Balance between Lytic and Lysogenic Viral Infection Is Driven by Trophic Status. Limnol. Oceanogr. 2013, 58, 465-474. [CrossRef]

8. Howard-Varona, C.; Hargreaves, K.R.; Abedon, S.T.; Sullivan, M.B. Lysogeny in Nature: Mechanisms, Impact and Ecology of Temperate Phages. ISME J. 2017, 11, 1511-1520. [CrossRef] [PubMed]

9. Canchaya, C.; Proux, C.; Fournous, G.; Bruttin, A.; Brüssow, H. Prophage Genomics. Microbiol. Mol. Biol. Rev. 2003, 67, 238-276. [CrossRef] [PubMed]

10. Silby, M.W.; Winstanley, C.; Godfrey, S.A.C.; Levy, S.B.; Jackson, R.W. Pseudomonas Genomes: Diverse and Adaptable. FEMS Microbiol. Rev. 2011, 35, 652-680. [CrossRef] [PubMed]

11. Knezevic, P.; Voet, M.; Lavigne, R. Prevalence of Pf1-like (pro)Phage Genetic Elements among Pseudomonas aeruginosa Isolates. Virology 2015, 483, 64-71. [CrossRef] [PubMed]

12. Rice, S.A.; Tan, C.H.; Mikkelsen, P.J.; Kung, V.; Woo, J.; Tay, M.; Hauser, A.; McDougald, D.; Webb, J.S.; Kjelleberg, S. The Biofilm Life Cycle and Virulence of Pseudomonas aeruginosa Are Dependent on a Filamentous Prophage. ISME J. 2009. [CrossRef] [PubMed]

13. Kuzio, J.; Kropinski, A.M. O-Antigen Conversion in Pseudomonas aeruginosa PAO1 by Bacteriophage D3. J. Bacteriol. 1983. [CrossRef]

14. Hayashi, T.; Baba, T.; Matsumoto, H.; Terawaki, Y. Phage-conversion of Cytotoxin Production in Pseudomonas aeruginosa. Mol. Microbiol. 1990. [CrossRef] [PubMed]

15. Webb, J.S.; Lau, M.; Kjelleberg, S. Bacteriophage and Phenotypic Variation in Pseudomonas aeruginosa Biofilm Development. J. Bacteriol. 2004, 186, 8066-8073. [CrossRef] [PubMed]

16. Secor, P.R.; Sweere, J.M.; Michaels, L.A.; Malkovskiy, A.V.; Lazzareschi, D.; Katznelson, E.; Rajadas, J.; Birnbaum, M.E.; Arrigoni, A.; Braun, K.R.; et al. Filamentous Bacteriophage Promote Biofilm Assembly and Function. Cell Host Microbe 2015. [CrossRef] [PubMed]

17. Zander, I. Identifying and Examining the Effect of Bacteriophage Derived Genes on Pseudomonas aeruginosa Virulence and Physiology. unpublished.

18. Novick, R. Properties of a Cryptic High-Frequency Transducing Phage in Staphylococcus aureus. Virology 1967. [CrossRef]

19. Wiederholt, K.M.; Steele, J.L. Prophage Curing and Partial Characterization of Temperate Bacteriophages from Thermolytic Strains of Lactococcus lactis ssp. Cremoris. J. Dairy Sci. 1993, 76, 921-930. [CrossRef]

20. Gay, P.; Le Coq, D.; Steinmetz, M.; Berkelman, T.; Kado, C.I. Positive Selection Procedure for Entrapment of Insertion Sequence Elements in Gram-Negative Bacteria. J. Bacteriol. 1985. [CrossRef]

21. Hmelo, L.R.; Hmelo, B.R.; Almblad, H.; Love, M.E.; Randall, T.E.; Tseng, B.S.; Lin, C.; Irie, Y.; Storek, K.M.; Yang, J.J.; et al. Precision-Engineering the Pseudomonas aeruginosa Genome with Two-Step Allelic Exchange. Nat. Rev. Drug Discov. 2016, 5, 1-8. [CrossRef]

22. Wozniak, D.J.; Ohman, D.E. Pseudomonas aeruginosa AlgB, a Two-Component Response Regulator of the NtrC Family, Is Required for AlgD Transcription. J. Bacteriol. 1991. [CrossRef] [PubMed] 
23. Zander, I.; Shmidov, E.; Roth, S.; Ben-David, Y.; Shoval, I.; Shoshani, S.; Danielli, A.; Banin, E. Characterization of PfiT/PfiA Toxin-antitoxin System of Pseudomonas aeruginosa That Affects Cell Elongation and Prophage Induction. Environ. Microbiol. 2020. [CrossRef]

24. Simon, R.; Priefer, U.; Puhler, A. A Broad Host Range Mobilization System for in Vivo Genetic Engineering: Transposon Mutagenesis in Gram Negative Bacteria. Nat. Biotechnol. 1983, 1, 784-791. [CrossRef]

25. Schweizer, H.P. Escherichia-Pseudomonas Shuttle Vectors Derived from PUC18/19. Gene 1991, 97, 109-112. [CrossRef]

26. Bankevich, A.; Nurk, S.; Antipov, D.; Gurevich, A.A.; Dvorkin, M.; Kulikov, A.S.; Lesin, V.M.; Nikolenko, S.I.; Pham, S.; Prjibelski, A.D.; et al. SPAdes: A New Genome Assembly Algorithm and Its Applications to Single-Cell Sequencing. J. Comput. Biol. 2012. [CrossRef] [PubMed]

27. Gurevich, A.; Saveliev, V.; Vyahhi, N.; Tesler, G. QUAST: Quality Assessment Tool for Genome Assemblies. Bioinformatics 2013. [CrossRef] [PubMed]

28. Rissman, A.I.; Mau, B.; Biehl, B.S.; Darling, A.E.; Glasner, J.D.; Perna, N.T. Reordering Contigs of Draft Genomes Using the Mauve Aligner. Bioinformatics 2009. [CrossRef]

29. Darling, A.E.; Mau, B.; Perna, N.T. Progressivemauve: Multiple Genome Alignment with Gene Gain, Loss and Rearrangement. PLoS ONE 2010. [CrossRef] [PubMed]

30. Arndt, D.; Grant, J.R.; Marcu, A.; Sajed, T.; Pon, A.; Liang, Y.; Wishart, D.S. PHASTER: A Better, Faster Version of the PHAST Phage Search Tool. Nucleic Acids Res. 2016, 44, W16-W21. [CrossRef] [PubMed]

31. Alikhan, N.F.; Petty, N.K.; Ben Zakour, N.L.; Beatson, S.A. BLAST Ring Image Generator (BRIG): Simple Prokaryote Genome Comparisons. BMC Genomics 2011. [CrossRef] [PubMed]

32. Mai-Prochnow, A.; Hui, J.G.K.; Kjelleberg, S.; Rakonjac, J.; McDougald, D.; Rice, S.A. “Big Things in Small Packages: The Genetics of Filamentous Phage and Effects on Fitness of Their Host". FEMS Microbiol. Rev. 2015, 39, 465-487. [CrossRef] [PubMed]

33. Euler, C.W.; Juncosa, B.; Ryan, P.A.; Deutsch, D.R.; McShan, W.M.; Fischetti, V.A. Targeted Curing of All Lysogenic Bacteriophage from Streptococcus pyogenes Using a Novel Counter-Selection Technique. PLoS ONE 2016, 11, e0146408. [CrossRef] [PubMed]

34. Ward, H.; Perron, G.G.; MacLean, R.C. The Cost of Multiple Drug Resistance in Pseudomonas aeruginosa. J. Evol. Biol. 2009, 22, 997-1003. [CrossRef] [PubMed]

35. Allen, J.P.; Ozer, E.A.; Minasov, G.; Shuvalova, L.; Kiryukhina, O.; Satchell, K.J.F.; Hauser, A.R. A Comparative Genomics Approach Identifies Contact-Dependent Growth Inhibition as a Virulence Determinant. Proc. Natl. Acad. Sci. USA 2020. [CrossRef] [PubMed]

36. Vasquez-Rifo, A.; Veksler-Lublinsky, I.; Cheng, Z.; Ausubel, F.M.; Ambros, V. The Pseudomonas aeruginosa Accessory Genome Elements Influence Virulence towards Caenorhabditis Elegans. Genome Biol. 2019. [CrossRef] [PubMed]

37. Karlsen, C.; Hjerde, E.; Klemetsen, T.; Willassen, N.P. Pan Genome and CRISPR Analyses of the Bacterial Fish Pathogen Moritella Viscosa. BMC Genom. 2017. [CrossRef]

38. Pasechnek, A.; Rabinovich, L.; Stadnyuk, O.; Azulay, G.; Mioduser, J.; Argov, T.; Borovok, I.; Sigal, N.; Herskovits, A.A. Active Lysogeny in Listeria monocytogenes Is a Bacteria-Phage Adaptive Response in the Mammalian Environment. Cell Rep. 2020. [CrossRef] [PubMed] 